\title{
REVISTA
}

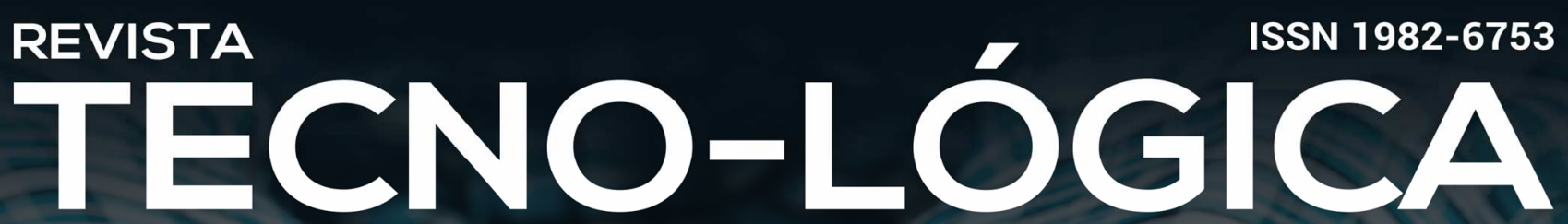

Edição Especial com publicação de trabalhos selecionados do V SIGEPRO

\section{ANÁLISE DA CAPACIDADE OPERACIONAL DE UM PROCESSO LOGÍSTICO FERROVIÁRIO ATRAVÉS DO ESTUDO DE TEMPOS E MOVIMENTOS}

Roberta Assis Costa ${ }^{1}$, Guilherme Silva Drumond ${ }^{1}$, Luigi Guarino Neto ${ }^{2}$, Fernanda Diniz Alves $^{3}$, Marcus Vinícius Damacena Silva ${ }^{*}$

1 Engenharia de Produção, Universidade Federal Fluminense, Volta Redonda, Brasil.

${ }^{2}$ Engenharia de Produção, Universidade de São Paulo, São Paulo, Brasil

3 Administração, Universidade Federal Fluminense, Volta Redonda, Brasil.

${ }^{4}$ Administração, Universidade Federal Rural do Rio de Janeiro, Volta Redonda, Brasil.

*E-mail: robertarsa@yahoo.com.br,

\section{RESUMO}

A crescente competitividade e a busca pela eliminação de desperdícios e redução de custos têm levado diversas organizações a avaliarem seus processos, a fim de maximizar a produtividade, aumentar sua eficiência operacional e garantir estabilidade no mercado. O presente trabalho fundamentou-se na metodologia do estudo de tempos e movimentos, buscando identificar a capacidade operacional de um processo logístico de carregamento ferroviário de produtos de aço. A aplicação da metodologia possibilitou avaliar sistematicamente a execução das atividades, permitindo identificar oportunidades de aumento de capacidade logística. Para alcançar os objetivos da pesquisa utilizou-se uma abordagem quantitativa e qualitativa, visando a partir da aplicação de cronometragens e observação, determinar o tempo padrão e a capacidade operacional, além de identificar oportunidades de eliminação de desperdícios. Os resultados mostram a eficácia na utilização da metodologia de tempos e movimentos contribuindo para aumento de produtividade e ganhos associados.

Palavras-chave: Estudo de tempos e movimentos. Capacidade produtiva. Tempo padrão.

\section{Introdução}

A competitividade crescente ao longo dos anos levou diversas organizações a aplicarem técnicas que otimizem seus processos, maximizando assim a produtividade a fim de alcançar

TECNO-LÓGICA, Santa Cruz do Sul, v. 24, n. nesp, p. 355-367, jul./dez. 2020 maiores níveis de eficiência operacional e funcionalidade no mercado em que estão inseridas.

Neste contexto, a adoção de técnicas e ferramentas como o estudo de tempos e movimentos, permitem a avaliação

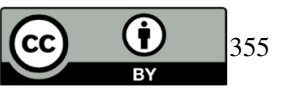




\section{REVISTA}

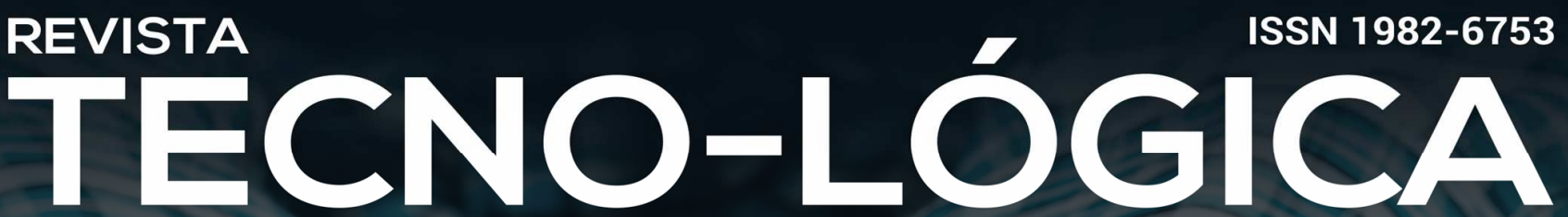

Edição Especial com publicação de trabalhos selecionados do V SIGEPRO

do comportamento dos processos fabris, a fim de determinar os melhores métodos de trabalho, garantir maior gestão das atividades e padronizar processos [1].

A utilização do estudo de tempos e movimentos permite a análise sistemática dos processos, a fim de identificar atividades que agregam valor e reduzir desperdícios com atividades que não agregam valor [2-3].

A aplicação das técnicas de tempos e movimentos permite também determinar a capacidade operacional da organização, pois avalia o volume ideal de produtos ou serviços que podem ser geridos em um espaço de tempo dentro de um nível adequado de atividades [4].

Neste contexto, o presente trabalho tem como objetivo avaliar a capacidade de escoamento ferroviário de um processo de carregamento de produtos de aço com aplicação do estudo de tempos e movimentos. Além disso, busca-se identificar oportunidades de aumento de produtividade e redução de desperdícios.

O processo logístico de escoamento ferroviário consiste no carregamento de produtos de aço, conhecidos como barras e fios máquina e ocorrem em três locais distintos de carregamento na empresa. A análise do processo logístico de carregamento é de suma importância, pois a falha na utilização do tempo disponível gera cobranças de multas por atraso de entrega, falhas no fluxo logístico, falhas nos prazos de entrega, além de restringir a capacidade produtiva almejada.

Os resultados da pesquisa apresentam o tempo padrão para a realização das atividades considerando fatores de tolerância anuentes ao processo. A análise das atividades a partir da determinação do tempo padrão, permite calcular a capacidade operacional atual do processo bem como as possibilidades futuras de expansão, garantindo maior atendimento aos clientes e melhor eficiência logística.

\section{Fundamentação Teórica}

\subsection{Estudo de Tempos e Movimentos}

A avaliação de processos fabris através de estudos de tempos e movimentos busca estabelecer padrões de trabalho para realização de atividades de forma mais eficiente, reduzindo desperdícios de tempos, materiais e recursos [1].

Os métodos sistemáticos de análise de processos produtivos foram idealizados por Frederick Taylor e por Frank e Lilian Gilbreth. As contribuições destes pioneiros foram fundamentais para a origem do tratamento adequado de dados e para melhorias dos métodos de trabalho [5].

Taylor foi o precursor da organização científica do trabalho e evidenciou em seus estudos que para obter melhores resultados seria fundamental o uso da investigação científica como método de estudo do trabalho, eliminando-se a subjetividade no processo produtivo. Nestes termos, a racionalização do trabalho, ou seja, a divisão de atividades em tarefas, seria possível através do estudo do tempo com aplicação de técnicas de cronometragem para determinar o tempo padrão para execução de uma atividade [6-7].

Nesta mesma perspectiva, o trabalho do casal Gilbreth corroborou para o conceito de organização científica do trabalho através da inclusão do elemento humano. O casal estudou aprofundadamente os métodos de execução de trabalho através da utilização da microfilmagem de movimentos, introduzindo o

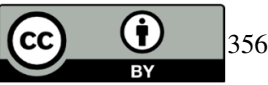




\section{REVISTA}

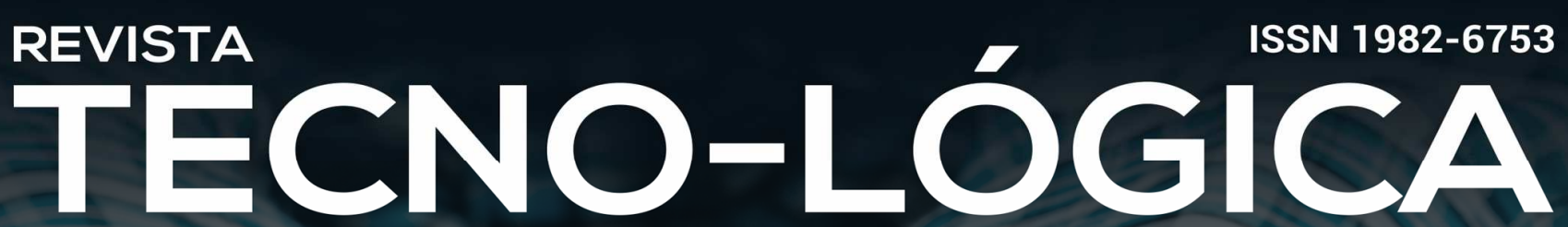

Edição Especial com publicação de trabalhos selecionados do V SIGEPRO

que é conhecido atualmente como estudo do método de trabalho [8]

As contribuições de Taylor com estudo do tempo e os Gilbreth com o estudo do método possibilitaram às organizações a padronização de rotinas e uso de técnicas para controle e análise de atividades, a fim de reduzir a ineficiência relacionada ao desperdício humano e melhor gestão de recursos no processo produtivo [9].

No âmbito industrial atualmente, a aplicação do estudo de métodos de trabalho possibilita identificar as atividades relevantes e determinar o tempo padrão para executá-las, assim como o método de execução, tal prática reduz desperdícios de processos, proporciona economia de tempo, recursos e energia, substituindo processos comuns por técnicas fundamentadas a partir da observação e experiência [10].

A utilização do estudo de tempos e movimentos permite a avaliação sistemática dos processos, possibilitando a partir da observação e cronometragem, a determinação do tempo padrão para realizar uma atividade. A aplicação deste método permite calcular a capacidade operacional para aumentar sua eficiência e produtividade [11].

Para realizar o estudo de tempos e movimentos é necessário mapear o processo através da divisão das atividades em tarefas. A partir da visão completa do processo faz-se necessária a realização de cronometragens preliminares para determinação do tamanho da amostra. Os dados obtidos com a análise dos tempos possibilitam identificar o tempo normal e o tempo padrão das atividades [12].

\subsubsection{Determinação do tamanho da amostra}

O estudo do comportamento de uma população exige certo grau de dedicação, observação e análise. A efetividade da avaliação pode ser observada a partir de inferências de uma amostra da população. Para que o número de amostras seja representativo, faz-se necessário calcular o número ideal de ciclos [13]. Para obter o tamanho da amostra (N) utiliza-se a equação 1:

$$
\mathrm{N}=\left(\frac{Z_{\alpha / 2} \cdot \sigma}{E r}\right)^{2}
$$

Onde:

$\mathrm{N}=\mathrm{n}^{\circ}$ de cronometragens a serem realizadas

$\mathrm{Z}=$ coeficiente de distribuição normal

$\sigma=$ desvio-padrão da amostra

$\mathrm{Er}=$ erro relativo da amostra

Para determinar o valor de $\mathrm{N}$, é necessário realizar uma cronometragem preliminar para obter a média da amostra e o desvio padrão. Após esta etapa, determina-se o valor do coeficiente de distribuição normal e o erro relativo aceitável para a amostra.

Para definir o coeficiente de distribuição normal, tornase necessário determinar o nível de confiança da amostragem. A

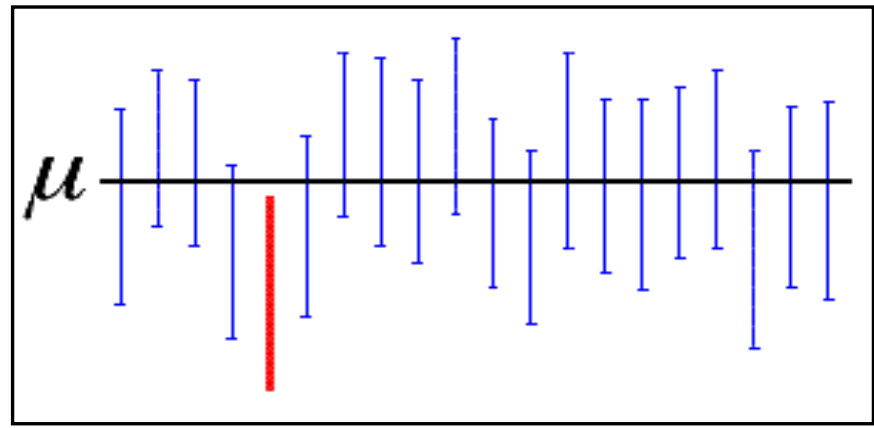

Figura 1 - Nível de Confiança [14].

A matéria publicada nesse periódico é licenciada sob forma de uma Licença Creative Commons - Atribuição 4.0 Internacional http://creativecommons.org//icenses/by/4.0/ 


\section{REVISTA}

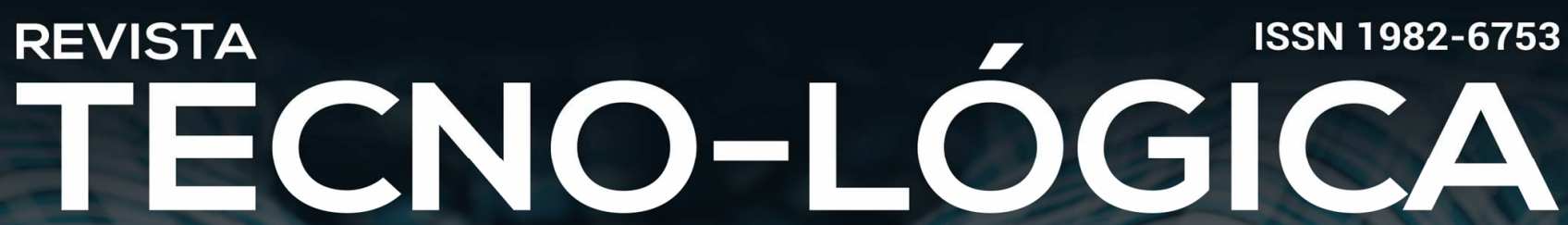

\section{Edição Especial com publicação de trabalhos selecionados do V SIGEPRO}

A linha horizontal apresenta a média populacional $(\mu)$. As linhas verticais representam intervalos de confiança que contêm a média populacional. Para um nível de confiança de 95\%, por exemplo, apenas 19 amostras de 20 produzem o intervalo de confiança contendo o parâmetro populacional. $\mathrm{O}$ nível de confiança, portanto, representa o percentual de intervalos no universo, que a partir do valor amostral representa o comportamento populacional [15-16].

É importante enfatizar que o tamanho da amostra, somente representa uma população se os dados da amostra resultarem em uma distribuição simétrica, ou seja, se a maior parte dos dados se concentrar próximo à média com pequena variação dos dados [17-18].

\subsubsection{Determinação do tempo normal}

O tempo em que um colaborador devidamente treinado leva para realizar sua atividade em um ritmo normal de trabalho é conhecido como tempo normal. Para calcular o tempo normal deve-se determinar o fator de velocidade ou ritmo do colaborador. A classificação do fator de velocidade é dada a seguir:
$\mathrm{V}=100 \%$ - velocidade normal de trabalho

V>100\% - velocidade acelerada

$\mathrm{V}<100 \%$ - velocidade lenta

O fator de velocidade é determinado pelo observador no momento da cronometragem e atribuído de forma subjetiva. Para calcular o tempo normal utiliza-se a equação 2 :

$$
T N=T C x V
$$

Onde:

$\mathrm{TN}=$ tempo normal

$\mathrm{TC}=$ tempo cronometrado

$\mathrm{V}=$ fator de velocidade ou ritmo

\subsubsection{Determinação do tempo padrão}

Para determinação do tempo padrão faz-se necessário conhecer as tolerâncias referentes ao processo. Estas tolerâncias consistem em concessões acrescentadas ao tempo utilizado de trabalho para permitir alivio à fadiga e necessidades pessoais [20-21]. O fator de tolerância pode ser calculado conforme a equação 3:

$$
\mathrm{FT}=\frac{1}{(1-P)}
$$

Onde:

$\mathrm{FT}=$ fator de tolerância

$\mathrm{P}=$ percentual de tempo de concessão

O tempo padrão trata-se do tempo utilizado para realizar determinada atividade considerando o ritmo de trabalho

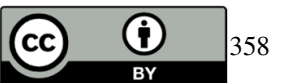




\section{REVISTA}

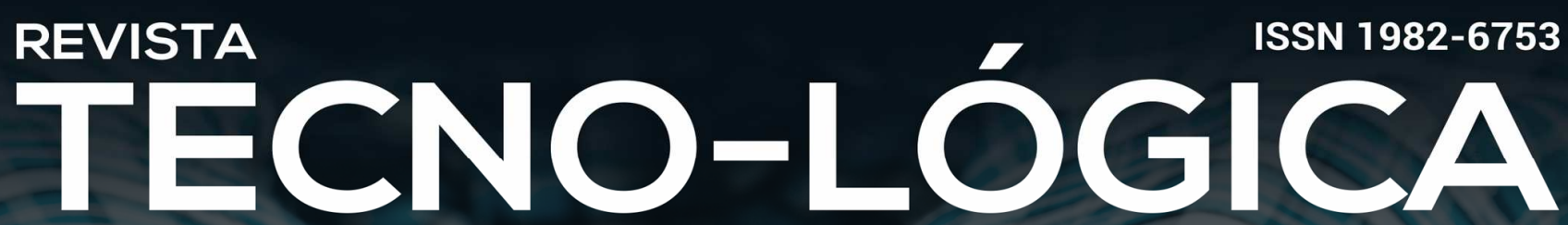

Edição Especial com publicação de trabalhos selecionados do V SIGEPRO

e as tolerâncias. Para determinar o tempo padrão utiliza-se a equação 4.

$$
T P=T N x F T
$$

Onde:

$\mathrm{TP}=$ tempo padrão

$\mathrm{TN}=$ tempo normal

FT = fator de tolerância

\subsubsection{Determinação da capacidade produtiva}

A capacidade produtiva representa a quantidade máxima que um processo pode produzir com os recursos disponível em um determinado período. O cálculo da capacidade produtiva é apresentado na equação 5 .

$$
C P=\frac{\text { Horas de Trabalho }}{T P}
$$

Onde:

$\mathrm{CP}=$ capacidade produtiva

TP = tempo padrão

A análise da capacidade produtiva está diretamente relacionada ao cálculo do tempo padrão de uma atividade realizada, portanto, deve ser avaliada após a análise do tempo gasto na operação através de técnicas de cronometragem [19].

\section{Metodologia}

A presente pesquisa consiste em um estudo de caso com aplicação de metodologia fundamentada em livros e artigos publicados no meio científico e acadêmico. A abordagem da pesquisa é quantitativa e qualitativa. A análise quantitativa foi realizada a partir da coleta de dados e utilização de técnicas estatísticas para determinar o comportamento do processo. Por outro lado, a abordagem qualitativa buscou por meio da compreensão dos fenômenos, analisar os procedimentos das atividades e identificar oportunidades de eliminar desperdícios.

O levantamento de dados foi realizado a partir de visitas técnicas ao local, observação e mapeamento dos processos com uso de fluxogramas. Como instrumento de pesquisa foram utilizados formulários de medição, desenvolvidos para o registro das cronometragens, além de cronômetros e câmeras de filmagem para avaliação dos tempos e métodos de trabalho.

A coleta de dados foi realizada em um período de 8 meses com observação de todas as atividades referentes ao carregamento ferroviário de barras e fios máquina que ocorrem em três áreas distintas da empresa: acabamento, laminação e estoque.

Com a base de dados das cronometragens, iniciou-se a elaboração dos cálculos para determinação do tempo padrão e cálculo da capacidade operacional.

\section{Resultados e discussões}

\subsection{Descrição do cenário}

O presente estudo apresenta a aplicação da metodologia de estudo de tempos e movimentos em um processo logístico de carregamento ferroviário de barras e fios máquina. O carregamento é realizado em 14 vagões que se distribuem em três locais de carregamento: acabamento, laminação e estoque. A distribuição dos vagões por local é mostrada na tabela 1.

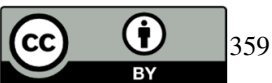




\section{REVISTA}

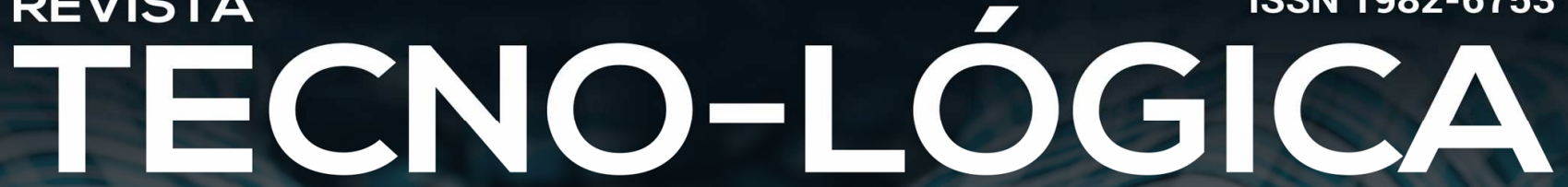

\section{Edição Especial com publicação de trabalhos selecionados do V SIGEPRO}

Tabela 1 - Vagões por local de carregamento

\begin{tabular}{lcc}
\hline LOCAL & PRODUTO & $\begin{array}{c}\text { NÚMERO } \\
\text { DE VAGÕES }\end{array}$ \\
\hline Acabamento & Barra & 2 \\
Laminação & Barra & 5 \\
Estoque & Fio máquina & 7 \\
\hline TOTAL & & $\mathbf{1 4}$ \\
\hline
\end{tabular}

O tempo disponível para executar a atividade sem pagamento de multa à concessionária ferroviária é de 36 horas. Portanto, faz-se necessário avaliar a capacidade produtiva dentro do período disponível para a execução do trabalho, a fim de não gerar multas e obter um processo com maior eficiência de logística.

\subsection{Descrição do processo}

O processo total consiste em seis etapas (figura2). No entanto, o presente estudo tem como objetivo avaliar apenas o processo de carregamento, cuja performance é de responsabilidade da logística interna. As demais etapas são realizadas por áreas parceiras.

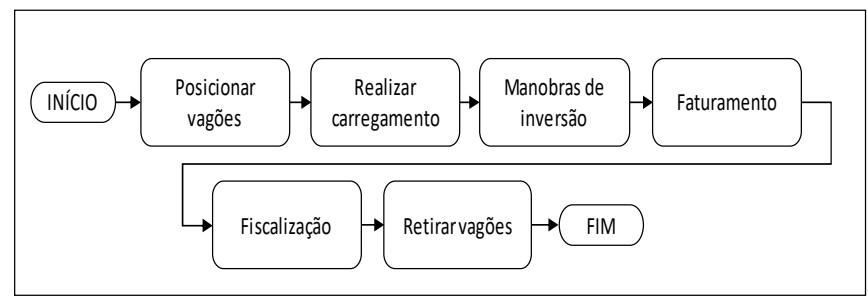

Figura 2 - Visão esquemática do processo

$$
\text { Os tempos médios para os processos de }
$$

posicionamento dos vagões, manobras de inversão, faturamento, fiscalização e retirada de vagões são apresentados na tabela 2 .
Tabela 2 - Tempos de atendimento (min) das áreas parceiras

\begin{tabular}{lc}
\multicolumn{1}{c}{ ATIVIDADE } & TEMPO \\
\hline Posicionamento dos vagões & 90 \\
Manobras de inversão & 60 \\
Faturamento & 240 \\
Fiscalização & 235 \\
Retirada dos vagões & 90 \\
\hline \multicolumn{2}{c}{ TOTAL } \\
\hline
\end{tabular}

As atividades relacionadas ao processo logístico de carregamento ferroviário são apresentadas na figura 3 .

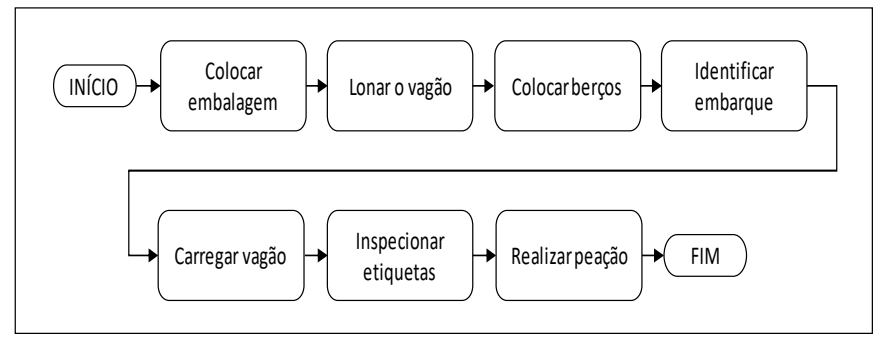

Figura 3 - Visão esquemática das atividades logísticas

$\mathrm{O}$ processo de carregamento engloba 7 etapas. A colocação de embalagem consiste na acomodação de madeira na superfície do vagão. O lonamento consiste na colocação de lona para proteção do produto e a colocação de berços permite a separação das cargas durante o posicionamento do material. A identificação do embarque consiste na marcação dos produtos a serem carregados durante a separação. O carregamento de vagão utiliza como recursos a ponte rolante no acabamento e laminação e uso de empilhadeiras no estoque. A inspeção da etiqueta consiste na coleta de informações através do coletor de dados. A peação é o processo de amarração de cargas que deve ser realizado no final do processo a fim de garantir a estabilidade da carga durante o trajeto. 


\section{REVISTA}

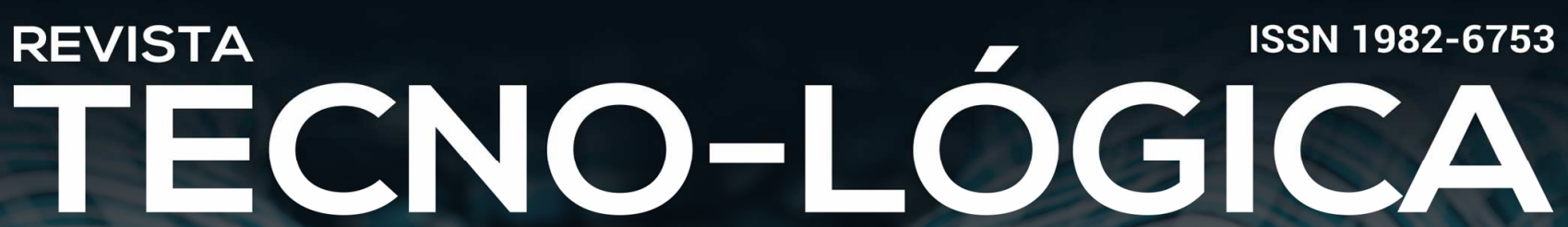

\section{Edição Especial com publicação de trabalhos selecionados do V SIGEPRO}

\subsection{Coleta de dados}

A atividade de coleta de dados iniciou-se com o mapeamento dos processos, a fim de determinar as atividades a serem cronometradas. As medições foram realizadas com utilização de cronômetro, formulário de coleta de dados e filmagens.

\subsubsection{Determinação do número de ciclos a serem} cronometrados.

Para determinação do número de cronometragens necessárias, foram realizadas cinco coletas preliminares em cada cenário, e definidos como parâmetro o nível de confiança de $85 \%$ e erro relativo de $15 \%$. A tabela 3 apresenta as cronometragens preliminares realizadas no acabamento.

A cronometragem preliminar mostrou que a média de tempo para o carregamento de um vagão no acabamento é de 121,64 minutos e o desvio-padrão é dado por 8,07 minutos.

Obtendo-se $\mathrm{N}$ igual a 15 cronometragens conforme equação 6.

$$
\mathrm{N}=\left(\frac{1,44 \times 8,07}{3,04}\right)^{2}=15
$$

$\mathrm{Na}$ tabela 4 são apresentadas as cronometragens preliminares do processo logístico de carregamento na área de laminação. A cronometragem preliminar mostrou que a média de tempo para o carregamento de um vagão na laminação é de 63,36 minutos e o desvio-padrão é dado por 5,23 minutos. Obtendo-se $\mathrm{N}$ igual a 15 cronometragens conforme equação 7.

$$
\mathrm{N}=\left(\frac{1,44 \times 5,23}{2,0}\right)^{2}=15
$$

$\mathrm{Na}$ tabela 5 são apresentadas as cronometragens preliminares do processo logístico de carregamento na área de estoque. A cronometragem preliminar mostrou que a média de tempo para o carregamento de um vagão no estoque é de 51,78 minutos e o desvio-padrão é dado por 5,32 minutos. Obtendo-se N igual a 25 cronometragens conforme equação 8.

$$
\mathrm{N}=\left(\frac{1,44 \times 5,32}{1,55}\right)^{2}=25
$$

\begin{tabular}{|c|c|c|c|c|c|c|c|}
\hline ETAPAS & T1 & $\mathbf{T 2}$ & T3 & $\mathbf{T 4}$ & T5 & Média & Desvio-padrão \\
\hline Colocar embalagem & 7,17 & 6,02 & 3,30 & 4,28 & 8,42 & & \\
\hline Lonar o vagão & 7,83 & 8,35 & 4,78 & 2,98 & 3,38 & & \\
\hline Colocar os berços & NA & NA & NA & NA & NA & & \\
\hline Identificar embarque & 24,92 & 20,00 & 11,47 & 10,00 & 20,00 & 121,64 & 8,07 \\
\hline Carregar o vagão & 53,72 & 61,65 & 79,22 & 75,55 & 82,50 & & \\
\hline Coletar e inspecionar etiquetas & 6,65 & 4,98 & 2,08 & 1,28 & 3,20 & & \\
\hline Realizar peação & 17,22 & 18,42 & 19,57 & 21,15 & 18,13 & & \\
\hline TOTAL & 117,50 & 119,42 & 120,42 & 115,25 & 135,63 & & \\
\hline
\end{tabular}

Tabela 3. Cronometragens preliminares no acabamento 


\section{REVISTA}

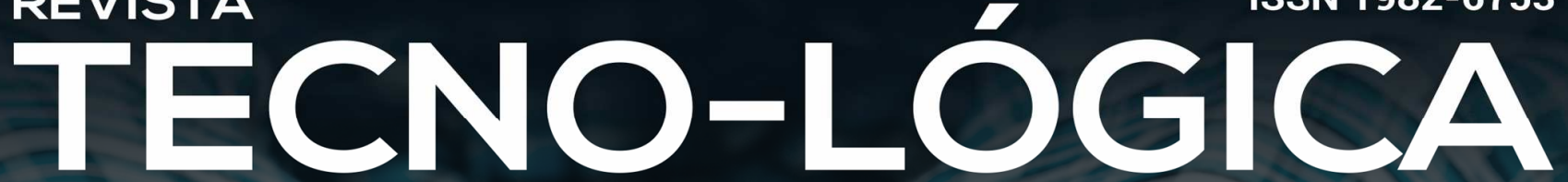

\section{Edição Especial com publicação de trabalhos selecionados do V SIGEPRO}

Tabela 4. Resultados das cronometragens preliminares na laminação

\begin{tabular}{|c|c|c|c|c|c|c|c|}
\hline ETAPAS & T1 & T2 & T3 & T4 & T5 & Média & Desvio-padrão \\
\hline Colocar embalagem & 3,00 & 3,65 & 2,32 & 3,05 & 2,60 & \multirow{7}{*}{63,36} & \multirow{7}{*}{5,23} \\
\hline Lonar o vagão & 3,18 & 2,98 & 2,90 & 5,60 & 1,68 & & \\
\hline Colocar os berços & NA & NA & NA & NA & NA & & \\
\hline Identificar embarque & 3,32 & 4,50 & 5,60 & 3,56 & 4,13 & & \\
\hline Carregar o vagão & 34,50 & 32,00 & 39,78 & 28,43 & 20,35 & & \\
\hline Coletar e inspecionar etiquetas & 1,02 & 4,08 & 1,57 & 1,80 & 2,03 & & \\
\hline Realizar peação & 20,12 & 17,70 & 17,33 & 19,43 & 24,57 & & \\
\hline Total & 65,14 & 64,92 & 69,50 & 61,87 & 55,36 & & \\
\hline
\end{tabular}

Tabela 5 - Resultados das cronometragens preliminares no estoque

\begin{tabular}{|c|c|c|c|c|c|c|c|}
\hline ETAPAS & T1 & $\mathbf{T 2}$ & T3 & T4 & T5 & Média & Desvio-padrão \\
\hline Colocar embalagem & NA & NA & NA & NA & NA & & \\
\hline Lonar o vagão & NA & NA & NA & NA & NA & & \\
\hline Colocar os berços & 4,82 & 5,45 & 3,50 & 2,32 & 1,23 & & \\
\hline Identificar embarque & 5,18 & 4,30 & 6,13 & 2,32 & 1,23 & 51,78 & 5,32 \\
\hline Carregar o vagão & 18,87 & 25,35 & 20,63 & 21,42 & 21,33 & & \\
\hline Coletar e inspecionar etiquetas & 2,67 & 3,43 & 3,48 & 3,75 & 3,67 & & \\
\hline Realizar peação & 20,97 & 21,72 & 16,13 & 16,00 & 23,00 & & \\
\hline Total & 52,51 & 60,25 & 49,87 & 45,80 & 50,47 & & \\
\hline
\end{tabular}

\subsection{Cálculo do fatro de tolerância}

Para determinar o fator de tolerância (FT), foi calculado o tempo disponível para refeições, necessidades pessoais, troca de turnos e fadiga, no período de 36 horas, conforme tabela 6 .

Tabela 6 - Tempo de concessão ao trabalhador (min)

\begin{tabular}{ll}
\hline CONCESSÕES & TEMPO \\
\hline Banheiro & 50 \\
Alimentação & 300 \\
Troca de turno & 75 \\
\hline TOTAL & 425
\end{tabular}

O tempo total concedido ao trabalhador considerando o período de 36 horas é de $425 \mathrm{~min}$. Sendo, portanto, o percentual permissivo (P) de 0,037. Com a determinação do tempo disponível de trabalho é possível calcular o fator de tolerância (FT), conforme Equação 9.

$$
\mathrm{FT}=\frac{1}{(1-0,037)}=1,04
$$

\subsection{Determinação do tempo padrão}

O tempo padrão (TP) é determinado a partir dos tempos cronometrados, da avaliação do tempo normal (TN), do fator de

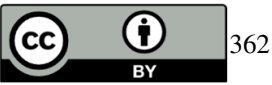




\section{REVISTA}

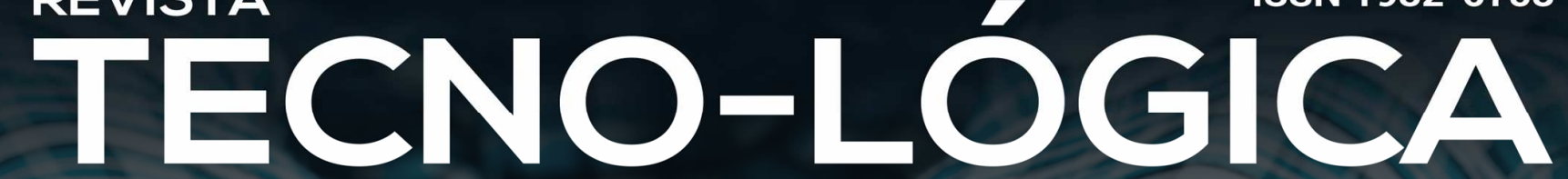

\section{Edição Especial com publicação de trabalhos selecionados do V SIGEPRO}

ritmo de trabalho $(\mathrm{V})$ e do fator de tolerância (FT). As tabelas 7,8 e 9 mostram os resultados do tempo padrão para cada área de carregamento.

Tabela 7 - Tempo normal e tempo padrão no acabamento

\begin{tabular}{lccccc}
\hline ETAPAS & MÉDIA & V & TN & FT & TP \\
\hline Colocar pernas de três & 5,84 & 1,10 & 6,42 & 1,04 & 6,68 \\
Lonar o vagão & 5,46 & 1,00 & 5,46 & 1,04 & 5,68 \\
Colocar os berços & NA & NA & NA & NA & NA \\
Identificar embarque & 15,26 & 0,90 & 13,73 & 1,04 & 14,28 \\
Carregar o vagão & 73,05 & 1,00 & 73,05 & 1,04 & 75,98 \\
Coletar e inspecionar & & & & & \\
etiquetas & 3,51 & 1,00 & 3,51 & 1,04 & 3,66 \\
Realizar peação & 19,99 & 0,90 & 17,99 & 1,04 & 18,71 \\
\hline TOTAL & 123,12 & 0,98 & 121,06 & 1,04 & 125,91 \\
\hline
\end{tabular}

O tempo padrão (TP) padrão para carregamento de barras no acabamento é de 125 min por vagão. A distribuição percentual dos tempos no acabamento é dada pela Figura 4.

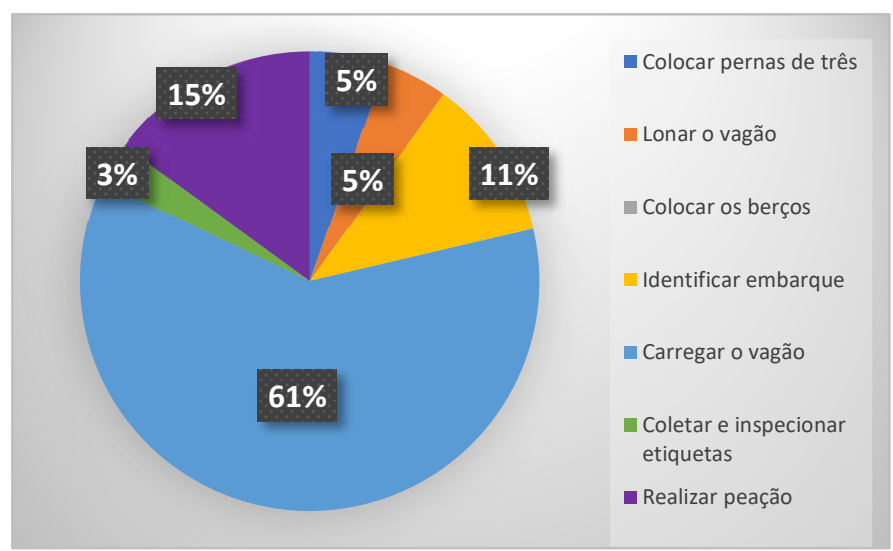

Figura 4 - Distribuição percentual de tempo no acabamento

A Figura 4 apresenta a estratificação percentual dos tempos, mostrando que os maiores tempos gastos ocorrem na etapa de carregamento do vagão (61\%), na peação (15\%) e na identificação do embarque (11\%). Neste processo foi possível observar que os tempos de carregamento são impactados pela concorrência de uso da ponte rolante e identificação manual dos embarques.

A tabela 8 apresenta os resultados do cálculo de tempo normal e padrão na laminação.

Tabela 8 - Tempo normal e tempo padrão na laminação

\begin{tabular}{lccccc} 
ETAPAS & MÉDIA & V & TN & FT & TP \\
\hline Colocar pernas de três & 2,94 & 1,00 & 2,94 & 1,04 & 3,05 \\
Lonar o vagão & 3,27 & 1,10 & 3,60 & 1,04 & 3,74 \\
Colocar os berços & NA & NA & NA & NA & NA \\
Identificar embarque & 4,36 & 0,90 & 3,93 & 1,04 & 4,08 \\
Carregar o vagão & 27,46 & 1,00 & 27,46 & 1,04 & 28,55 \\
Coletar e inspecionar & & & & & \\
etiquetas & 2,55 & 1,10 & 2,81 & 1,04 & 2,92 \\
Realizar peação & 19,74 & 1,00 & 19,74 & 1,04 & 20,53 \\
\hline Total & 60,17 & 1,00 & 60,17 & 1,04 & 62,58
\end{tabular}

O tempo padrão (TP) padrão para carregamento de barras na laminação é de 62,58 min por vagão. A distribuição percentual dos tempos na laminação é dada pela Figura 5.

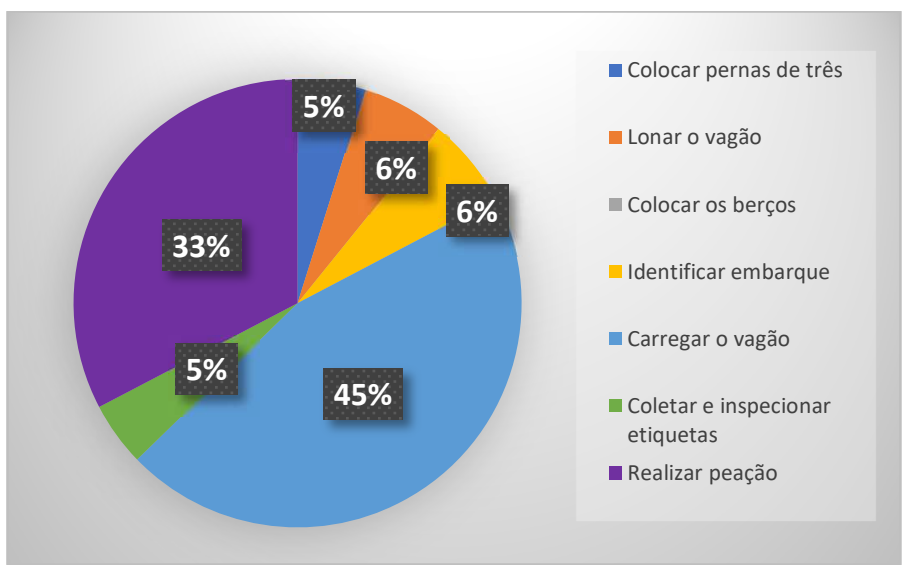

Figura 5 - Distribuição percentual de tempo na laminação 


\section{REVISTA}

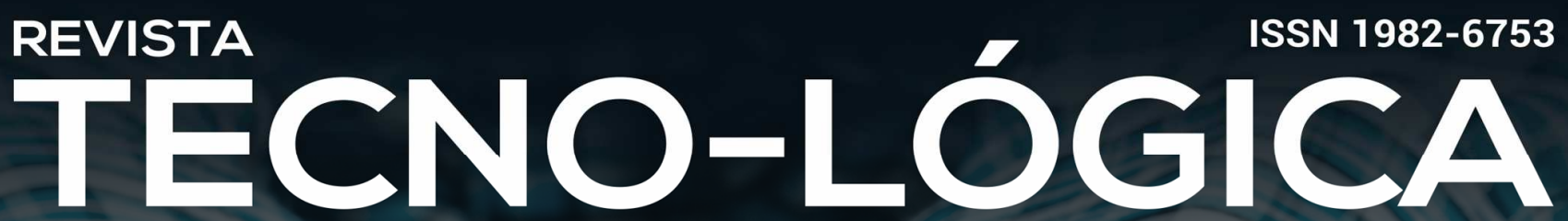

\section{Edição Especial com publicação de trabalhos selecionados do V SIGEPRO}

A Figura 5 apresenta a estratificação dos dados, mostrando que os maiores tempos gastos ocorrem no carregamento do vagão (45\%) e na peação $(33 \%)$. Neste processo foi possível observar que os tempos de carregamento são impactos pela concorrência de pontes rolantes, gerando paradas constantes para realização de outras atividades.

A tabela 9 apresenta os resultados do cálculo de tempo normal e padrão no estoque.

Tabela 9 - Tempo normal e tempo padrão no estoque

\begin{tabular}{lccccc}
\hline ETAPAS & MÉDIA & V & TN & FT & TP \\
\hline Colocar pernas de três & NA & NA & NA & NA & NA \\
Lonar o vagão & NA & NA & NA & NA & NA \\
Colocar os berços & 2,86 & 1,00 & 2,86 & 1,04 & 2,98 \\
Identificar embarque & 5,92 & 0,90 & 5,33 & 1,04 & 5,54 \\
Carregar o vagão & 26,12 & 1,00 & 26,12 & 1,04 & 27,16 \\
Coletar e inspecionar & & & & & \\
etiquetas & 3,26 & 1,00 & 3,26 & 1,04 & 3,39 \\
Realizar peação & 25,36 & 1,00 & 25,36 & 1,04 & 26,38 \\
\hline Total & 63,52 & 0,98 & 62,25 & 1,04 & 64,74 \\
\hline
\end{tabular}

O tempo padrão (TP) padrão para carregamento de fios máquina no estoque é de 64,74 min por vagão. A distribuição percentual dos tempos no estoque é dada pelo Figura 6.

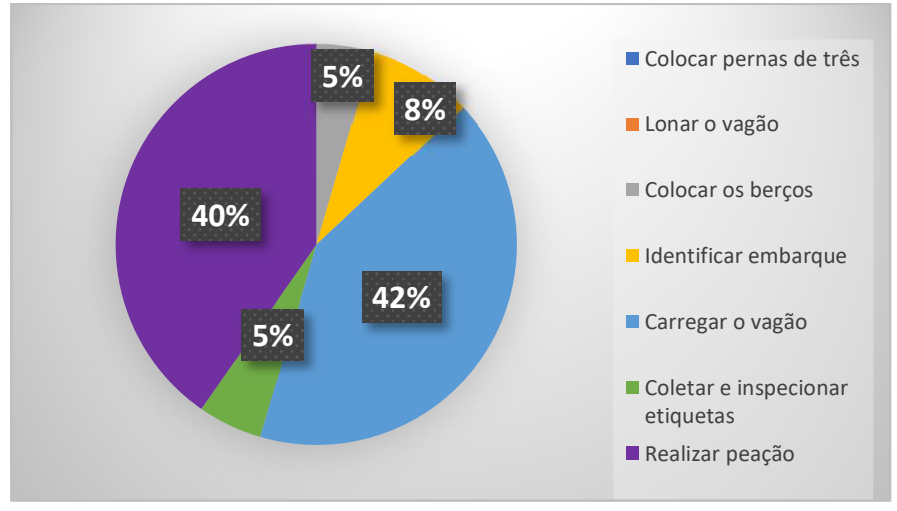

Figura 6 - Distribuição percentual de tempo no estoque
A Figura 6 apresenta a estratificação dos dados, mostrando que os maiores tempos gastos ocorrem no carregamento do vagão (42\%) e peação (40\%). Neste caso, não se observou interferências para realização da atividade.

Com a análise do tempo padrão por área de carregamento e considerando o total de vagões carregados em 36 horas é possível determinar o tempo padrão total de carregamento de vagões, conforme apresentando na tabela 10.

Tabela 10 - Tempo padrão total para o processo de carregamento

\begin{tabular}{cccc}
\hline $\begin{array}{c}\text { LOCAL DE } \\
\text { CARREGAMENTO }\end{array}$ & $\begin{array}{c}\text { TEMPO } \\
\text { PADRÃO } \\
\text { POR VAGÃo }\end{array}$ & $\begin{array}{c}\text { TOTAL DE } \\
\text { VAGÕES }\end{array}$ & $\begin{array}{c}\text { TEMPO } \\
\text { PADRÃO } \\
\text { TOTAL }\end{array}$ \\
\hline Acabamento & 125,70 & 2 & 251,40 \\
Laminação & 62,48 & 5 & 312,39 \\
Estoque & 64,63 & 7 & 452,43 \\
\hline & & TOTAL (min) & $\mathbf{1 0 1 6 , 2 2}$ \\
\hline
\end{tabular}

O tempo total padrão para o carregamento dos 14 vagões é de 1016,22 min ou 16,94 horas. A Figura 7 mostra a distribuição de tempo para o escoamento ferroviário dos produtos de aço.

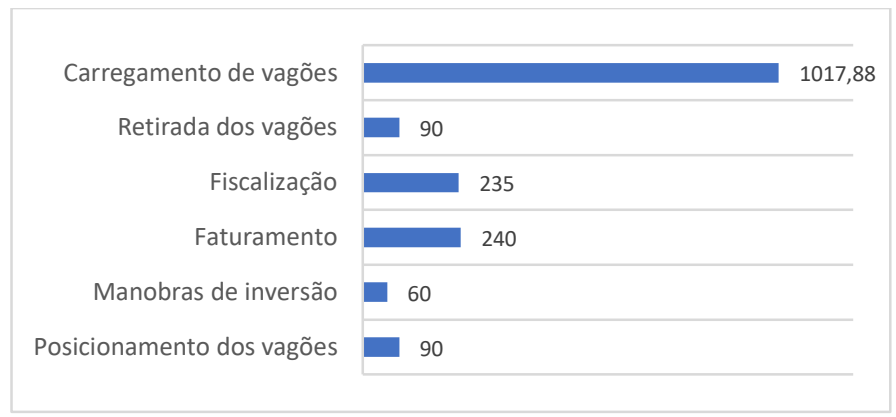

Figura 7 - Distribuição de tempos das atividades de escoamento ferroviário (min)

A Figura 7 mostra a estratificação dos tempos no processo logístico. É possível observar que o maior tempo está relacionado ao carregamento de vagões pela logística interna. 


\section{REVISTA}

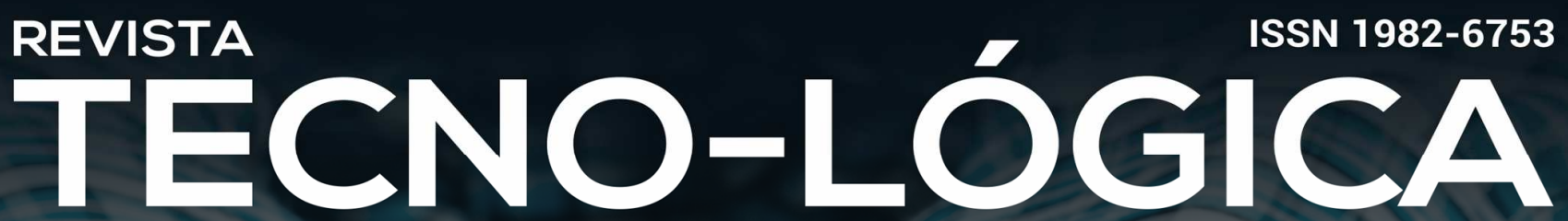

Edição Especial com publicação de trabalhos selecionados do V SIGEPRO

\subsection{Cálculo da Capacidade Operacional}

Considerando-se o tempo acordado com as áreas parceiras e o tempo padrão determinado para realizar o carregamento dos 14 vagões, é possível determinar o tempo total dedicado ao processo logístico de escoamento conforme tabela 11.

Tabela 11 - Tempo total atual (min)

\begin{tabular}{lc}
\hline \multicolumn{1}{c}{ ATIVIDADE } & TEMPO \\
\hline Posicionamento dos vagões & 90 \\
Manobras de inversão & 60 \\
Faturamento & 240 \\
Fiscalização & 235 \\
Retirada dos vagões & 90 \\
Carregamento de vagões & 1017,88 \\
\hline \multicolumn{1}{c}{ TOTAL } & $\mathbf{1 7 3 2 , 9}$
\end{tabular}

Por fim, calcula-se a capacidade operacional do processo considerando o tempo disponível de 36 horas ou 2160 minutos, excluindo o tempo acordado com as áreas parceiras (715 minutos) dividido pelo tempo padrão médio de carregamento $(72,71$ minutos), conforme equação 10 .

$$
\mathrm{CP}=\frac{2160 \min -715 \min }{72,71 \min }=19
$$

O resultado do cálculo da capacidade produtiva mostra que há um potencial para carregar adicionalmente 5 vagões, ou seja, a capacidade operacional no tempo disponível de 36 horas é de 19 vagões.

\section{Conclusões}

Esta pesquisa apresentou a avaliação do processo de carregamento logístico ferroviário de produtos de aço, como barras e fios máquina, distribuídos em três áreas de carregamento, possibilitando identificar a capacidade operacional atual e identificar oportunidades. O estudo apresenta detalhadamente a aplicação da metodologia de tempos e movimentos, considerando a realização de cronometragens preliminares para determinação do tamanho da amostra $(\mathrm{N})$ e posterior determinação dos tempos normais $(\mathrm{TN})$, fatores de velocidade $(\mathrm{V})$, fatores de tolerância (FT) e por fim, a determinação do tempo padrão (TP). A efetividade da avaliação dos parâmetros permitiu analisar de modo sistemático o comportamento do processo.

$\mathrm{O}$ estudo de tempos e movimentos mostrou a diferença entre os tempos de processo nos locais de carregamento, principalmente na área de acabamento, que possui o dobro de tempo para realização da atividade quando comparado aos demais processos. Esta diferença foi evidenciada pela observação de interferência nos processos, concorrência entre equipamentos e realização de atividades manuais. Diferentemente do que ocorre nas áreas de laminação e estoque em que a identificação de embarque é automática, foi evidenciada que no acabamento esta atividade é realizada manualmente, impactando no tempo de processo. Desta forma, é possível identificar oportunidades para maximizar a produtividade a partir da implementação da automatização da atividade.

Os resultados da pesquisa mostram-se satisfatórios, uma vez que o estudo permitiu determinar a capacidade produtiva atual. Observa-se que o tempo disponível para a carga de 14 vagões é suficiente para realização da atividade, não havendo necessidade de reorganização do processo, contratação de novos funcionários ou obtenção de novos equipamentos. Verificou-se também que é possível dentro do tempo disponível carregar até 5 vagões adicionais de produtos de aço, totalizando 19 vagões. $O$ estudo mostra a eficácia do método para o cálculo da capacidade

A matéria publicada nesse periódico é licenciada sob forma de uma Licença Creative Commons - Atribuição 4.0 Internaciona http://creativecommons.org/licenses/by/4.0/ 


\section{REVISTA}

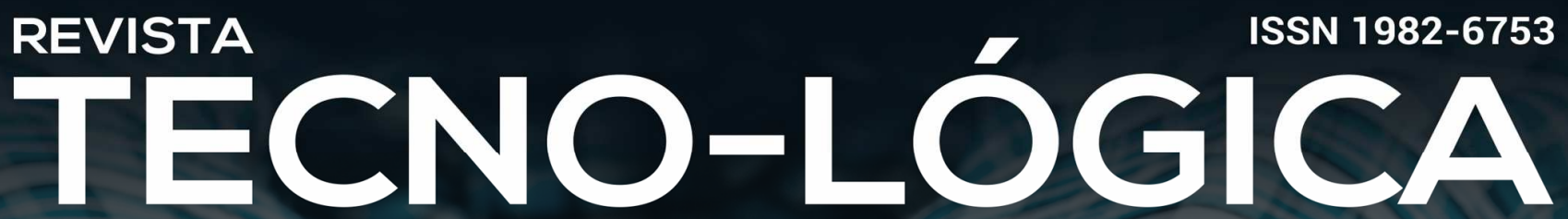

Edição Especial com publicação de trabalhos selecionados do V SIGEPRO

operacional considerando métricas pré-definidas e disponibilidade de equipamentos e mão de obra.

Por fim como próximos passos, propõe-se um estudo aprofundado para realização do carregamento de 19 vagões considerando métricas de tempo já abordadas no presente estudo, além da avaliação das capacidades das linhas férreas e impacto no ciclo de escoamento logístico com a adição dos novos vagões.

\section{TÍTULO EM INGLÊS}

\section{ANALYSIS OF THE OPERATIONAL CAPACITY OF A \\ RAILWAY LOGISTIC PROCESS THROUGH THE STUDY OF TIMES AND MOVEMENTS}

\section{ABSTRACT:}

The increasing competitiveness and the search for the elimination of waste and cost reduction have led several organizations to evaluate their processes, in order to maximize productivity, increase their operational efficiency and guarantee stability in the market. The present work was based on the methodology of the study of times and movements, seeking to identify the operational capacity of a logistical process of railroad loading of steel products. The application of the methodology made it possible to systematically evaluate the execution of activities, allowing to identify opportunities for increasing logistical capacity. To achieve the research objectives, a quantitative and qualitative approach was used, aiming from the application of timings and observation, to determine the standard time and operational capacity, in addition to identifying opportunities for eliminating waste. The results show the effectiveness in the use of the methodology of times and movements contributing to increase of productivity and associated gains.
Keywords: Study of times and movements. production capacity. standard time.

\section{Referências}

[1] NEGREIROS, R.F., LIMA, R. N. Projeto de engenharia de métodos numa fábrica de sorvetes. Simpósio de Engenharia de Produção da Região Nordeste, 2011.

[2] BARNES, R.M. Estudo de movimentos e de tempos: projeto e medida do trabalho. São Paulo: Edgard Blücher, 1977.

[3] CURY, P.H.A, SARAIVA, J. Time and motion study applied to a production line of organic lenses in Manuas Industrial Hub. Gest.Produção, São Carlos, 2018.

[4] SLACK, N., CHAMBERS, S., JOHNSTON, R. Administração da produção. São Paulo: Atlas, 2009.

[5] FERREIRA, L.A.F., SANTOS, A.C.S.G., DIAS, J.O., PESSANHA, L.P.M., Engenharia de métodos: uma revisão de literatura sobre o estudo de tempos e métodos. Revista Fatec, Zona sul, 2018.

[6] SOUSA, S.R.O., LACERDA, M.S., LAMEIRA, I.W.A., CAMPOS, E.M.R., OLIVEIRA, R.D. O estudo de tempos e movimentos na eliminação de desperdícios: uma aplicação prática na área de limpeza industrial mecanizada. Revista Exata, São Paulo, 2017.

[7] VIJAI, J. P. Relevance of Taylor's principles to modern shop-floor practices: a benchmarking work study. Benchmarking: An International Journal, , 2017.

[8] BALAR, M.J.H., RATHOLD, M.H.A., SHAH, M.R. A review on effective time and motion study on construction project: "a case study of surat city". International Journal for Scientific research development, 2018.

[9] LOPETEGUI, M. et al. Time motion studies in healthcare: What are we talking about? Journal of Biomedical Informatics, 2014.

[10] PEINADO J., GRAEML, A. R. Administração da produção. Curitiba: Unicenp, 2007.

[11] MAGU, P., KHANNA, K., SEETHARAMAN, P. Path process chart: a technique for conducting time and motion study. Elsiever, India, 2015.

[12] CORDEIRO, B.A.F., BARREIROS, E.C.M., RANIERI, J.R., RODRIGUES, N.A.C., FERREIRA, V.T.S. Estudo de tempos e movimentos em uma empresa de construção civil em Belém: um estudo para padronização da etapa de escoamento. EPAEP, 2015.

A matéria publicada nesse periódico é licenciada sob forma de uma Licença Creative Commons - Atribuiç̃ 4.0 Internacional http://creativecommons.org/licenses/by/4.0/ 


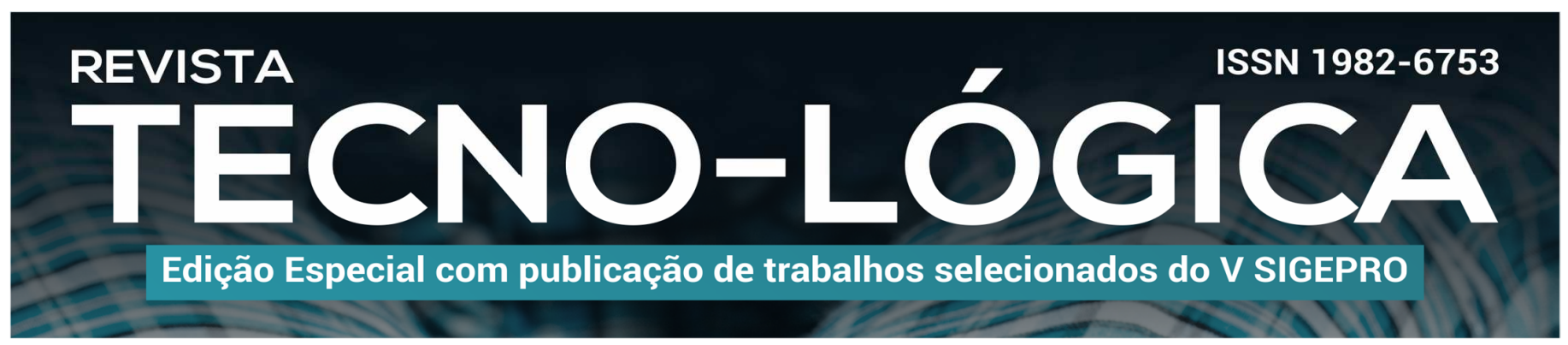

[13] MOURÃO JR, C. A. Questões em bioestatística: o tamanho da amostra. Revista Interdisciplinar de Estudos Experimentais, Juiz de Fora, 2009

[14] COSTA, A.F.B.; EPPRECHT, E.K.; CARPINETTI, L.C.R. Controle estatístico da qualidade. São Paulo: Editora Atlas, 2008.

[15] TRIOLA, Mário F. Introdução à Estatística. 7a. Ed. Rio de Janeiro: LTC, 1999.

[16] ARAÚJO, L.C.G. Organizações, sistemas e métodos e as novas tecnologias de gestão organizacional. São Paulo: Atlas, 2006.

[17] SANTOS, A.G, NETO, H.C.A., LACERDA, E.F., LUNA, W.A., FURLANETTO, E.L. A importância dos gráficos de controle para monitorar a qualidade dos processos industriais: estudo de caso numa indústria metalúrgica. ENEGEP. Salvador, 2009.

[18] MONTGOMERY, D.C; RUNGER, G.C. Estatística aplicada e probabilidade para engenheiros. Rio de Janeiro: LTC.Editora, 2012.

[19] GAITHER, N.; FRAZIER, G. Administração da produção e operações. 8 Ed. São Paulo:Thomson, 1999.

[20] BARboSA, S.N.S., CAVAlCANTE, B.G.L., SILVA, F.F., NEGRÃO, L.L.L., SANTOS, L.O. Studies of time: analysis of the production capacity of the operation of the production of picoles. Revista Latino-Americana de Inovação e Engenharia de Produção, 2017.

[21]STIVAL, S.W., BARBOSA, T.D., ALENCAR, A.C.L., DIAS, R.S., MOURA, R.R. Estudo de tempos e movimentos: análise do processo produtivo na confecção de camisetas. SIENPRO. Universidade Federal de Goiás, Goiás, 2015. 